\title{
Machine learning method to ensure robust decision-making of AVs
}

\author{
Ramdane Tami ${ }^{1}$, Boussaad Soualmi ${ }^{2}$, Abdelkrim Doufene ${ }^{2}$, Javier Ibanez ${ }^{3}$, and Justin Dauwels ${ }^{4}$
}

\begin{abstract}
Replacing the human driver to perform the Dynamic Driving Task (DDT)[1] will require perception, complex analysis and assessment of traffic situation. The path leading to success the deployment of fully Autonomous Vehicle (AV) depends on the resolution of a lot of challenges. Both the safety and the security aspects of $\mathrm{AV}$ constitute the core of regulatory compliance and technical research. The Autonomous Driving System (ADS) should be designed to ensure a safe manoeuvre and a stable behaviour despite the technological limitations, the uncertainties and hazards which characterize the real traffic conditions. In fully Autonomous Driving situation, detecting all relevant objects and agents should be sufficient to generate a warning, however the ADS requires further complex data analysis steps to quantify and improve the safety of decision making. This paper aims to improve the robustness of decision-making in order to mimic human-like decision ability. The approach is based on machine learning to identify the criticality of the dynamic situation and enabling ADS to make appropriate decision and fulfil safe manoeuvre.
\end{abstract}

\section{INTRODUCTION}

In movies and the literature world, many fictions about the future deal with the coexistence of robots and humans. The autonomous vehicle could be one of those robots which could characterize the future. Although in several application fields, the machines have already successfully replaced some human tasks, the integration of autonomous vehicles in the traffic environment will be the first large scale and complex coexistence scenario. This topic worries the public

This work was supported by IRT-SystemX and ERIAN

${ }^{1} \mathrm{R}$. Tami is with Energy Research Institute, Nanyang Technological University, Singapore 639798 rtami antu. edu. s g

${ }^{2}$ B. Soualmi and A. Doufene are with the Institut de Recherche Technologique SystemX, 8, avenue de la Vauve 91127 PALAISEAU CEDEX, France boussaad. soualmidirt-systemx. fr, abdelkrim. doufenedirt-systemx.fr

${ }^{3} \mathrm{~J}$. Ibanez is with Renault S.A.S, Technocentre Renault, 1 Avenue du Golf, 78280 Guyancourt, France javier.ibanez@renault. fr

4 J. Dauwels is with the School of Electrical and Electronic Engineering and the School of Physical and Mathematical Sciences, Nanyang Technological University, Singapore 639798 jdauwelsentu.edu.sg and the deployment will require to rethink the legislation and the regulation. Despite the interest expressed by governments, manufacturers and X-tier companies, driving in the urban context still escapes being fully autonomous because of numerous challenges and constraints. It is still unclear when ADSs will be mature enough to replace completely the human in performing the DDT.

The purpose of deploying AV necessitates advanced technologies particularly in term of communication and it also requires a global regulation alignment. On the one hand, this requires to define the operating and regulatory frameworks. On the other hand, it requires to develop human-like driving strategies [2], and a panoply of intelligent systems in order to have AV behaviour similar to that of a human who has spent his whole life continuously learning how to assess risks and make appropriate decisions.

From a regulatory point of view, ADSs require to address safety and security. OEMs need to provide evidence that the $\mathrm{AV}$ is at leats safe as a human driven vehicle. According to World Health Organization (WHO), in the last decade, 1.3 million people have died in road traffic accidents and millions of people get injured in accidents caused by human drivers errors every year. Concretely the most accurate way to assess the safety of AVs is to test-drive them in real traffic and observe their performance. However a distance-based validation would require driving millions of kilometres in order to attain a statistical significance about the system efficiency and capabilities [3].

Although, public road testing and dedicated testtrack testing are mandatory to verify real condition driving, designing virtual test framework which does not endanger the road users seems an appropriate and plausible alternative to the distance-based approach which is neither economical nor suitable for repeatable test. Therefore reproducing traffic environment in simulator with fair fidelity can have complementary role in reducing time and cost required by $\mathrm{AV}$ validation process with repeatable test runs.

For example in the urban context, since numerous and different types of agents have mingled dynamics, 
the ADSs requires a very robust perception system and decision making system. First, the sensors should be sensitive and suitable for an accurate real-time acquisition and communication. Then, the reliability of AV decision and action is a prerequisite to AV deployment in complex and time-varying traffic contexts.

Different manoeuvres and actions are jointly realized by human drivers and Advanced Driver Assistance Systems (ADAS) to avoid critical and risky driving situations, by doing basic actions such as: steering, braking, acceleration and combination of previous actions. The biggest challenge for $\mathrm{AVs}$ is the development of efficient approaches enabling to completely replace the driver decision-making while preserving the safety.

$\mathrm{AV}$ is driverless, then the human decision role is removed. Even without driver the ADS should be able to assess the complexity of the DDT according to agents behaviour and make decisions. This paper addresses a machine learning based approach for decision making in order to ensure safe manoeuvres in a driverless context.

The section II summarizes the state of the art of technologies related to AVs with a focus on the decision making during manoeuvre. The section III discusses risk assessment and introduces Adaboost algorithm. The section IV describes the proposed framework and shows the obtained results. A summary and conclusion are given in the section $\mathrm{V}$.

\section{RELATED WORK}

Autonomous vehicle depends on five technological locks (Fig.1): perception, localization, path planning, vehicle control, and decision making [4]. The latter system makes a choice after comparing several possibilities based on the data given by other systems, such as current vehicle state, sensors, weather conditions and traffic signs. An appropriate command is provided enabling safe movement. Then, an ADS should be able to prevent road traffic accidents and mitigate their consequences which implies new functional safety challenges.

During the DDT to arrive to his intended destination, the human driver behaves and reacts in order to avoid hazards and comply with the traffic rules. However, to achieve a fully driverless state, the AV technology will require human-like capabilities for the control and the decision making in order to move safely and smoothly in mixed and varying environment characterized by uncertainties, disturbances and complex traffic scenarios.

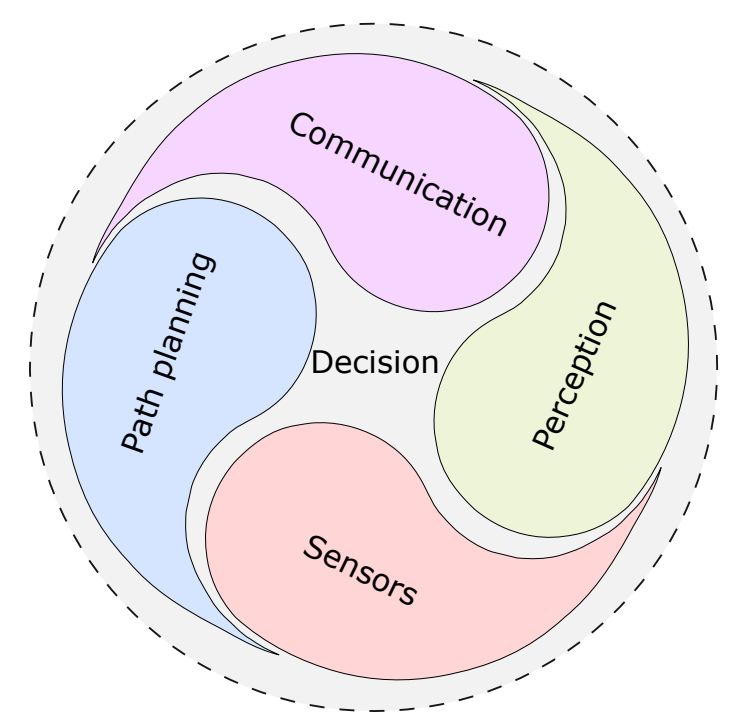

Fig. 1: Challenges and requirements for autonomy.

Moreover managing the risk of false alarms is another challenge to avoid collisions.

To ensure an optimal design and robustness of AV decision making systems, developing algorithms which monitor the vehicle safety represents a key point of ADSs research. Currently, deep research and development activities are carried out world wide [5], [6] and [7]. Algorithm development activities are gradually increasing because of the critical challenges that need to be addressed. For example in case of path planning issue, [8] have proposed a robust lane change approach. The work is motivated by the discontinuous availability of valid data from sensors. [9] have addressed a problem of smoothness in lane change. The approach is based on fuzzy-neural network and genetic algorithm.

Furthermore, human-like decision making in AV [2] is an emerging field where numerous relevant and promising methods are under development. [10] present an overtaking system for AV. The approach uses fuzzy controller to mimic human behaviour and reactions during overtaking manoeuvres. [11] conducted research on the path planning problem in dynamic environment. They proposed a Binary Decision Diagram (BDD) approach using a set of parametrized candidate trajectories. [12], proposed an architecture of two layers describing a mathematical coupling of vehicle and driver within an intersection scenario. The article used hidden Markov models to estimate Human driver behaviour. [13] presented a decision approach based on hybrid structure formed by a model of predictive controller and feed-forward neural networks to teach a vehicle how to drift like professional drivers do. In the same context, 
a hierarchical reasoning game theory-based approach has been presented to test and verify the autonomous driving algorithms [14]. In such systems, generating Trust index, based on partial or local data, could help to evaluate the decision-making [15].

Subsequently, some improvement have been introduced to deal with uncertainty. [16] pointed out that the predictive multi-criteria decision-making approach based on fuzzy logic can handle successfully uncertain data.

This paper does not address sensing or communication issues. We assume that the input data are available, we can propose a learning anomaly detection framework that goes beyond the current state-of-theart by considering not only the prediction of critical situation but also the decision under constraints.

\section{PROBLEM FORMULATION}

Human drivers use a variety of biological sensors when performing the DDT. We use both eyes for perception and our brain as well as our intuition for reasoning and decision. These capabilities enable to analyse the environment and react in less than $1 \mathrm{~s}$, then the safety is achieved as far as possible. But in a driverless state, the capabilities of AV to deal safely with the urban traffic depends on ADS abilities to handle a multi-disciplinary challenges [17].

\section{A. Driving risk assessment}

Human driver analyses the environment before the manoeuvre decision, whereas in $\mathrm{AV}$ the total control is transferred from Human to ADS. Therefore monitoring processes which analyse the risk, predict movements and deduce the intention of the other agents is required. In this case, the classification and the study of the dynamics of the other agents can be used to evaluate and estimate the risk and the possibility of collision before action.

[18] introduced the concept of traffic pressure mode and proposed a driving mode decision in the case of lane change. It is based on the assessment and monitoring of rear and front safety distance zones [19].

Still, efforts to bring these approaches to a level of human-like abilities is linked to managing transition state, movement intention and hesitation of the agents. These aspects which are usually managed by intuition may constitute a limitation for a deterministic assessment criteria.

Let us consider $m_{\text {most }_{a} p p r} \in M_{\text {all }}$ the most appropriate decision before execution of a driving manoeuvre based on its feasibility and the safety. The safety is based on traffic conditions and the road traffic rules. The feasibility is function of dynamics, perception and road configuration. The manoeuvre risk evaluation is mainly related to the relative speed, and the relative distance between agents:

$$
R=R_{T T C}+R_{T I V},
$$

where $T T C$ and $T I V$ are time to collision and intervehicle time, respectively. $R_{T T C}$ and $R_{T I V}$ are the risk associated with TTC and TIV respectively [20].

$$
R_{T I V}=f\left(P_{T I V}\right),
$$

where $P_{T I V}$ denotes the possibility of collision associated to TIV.

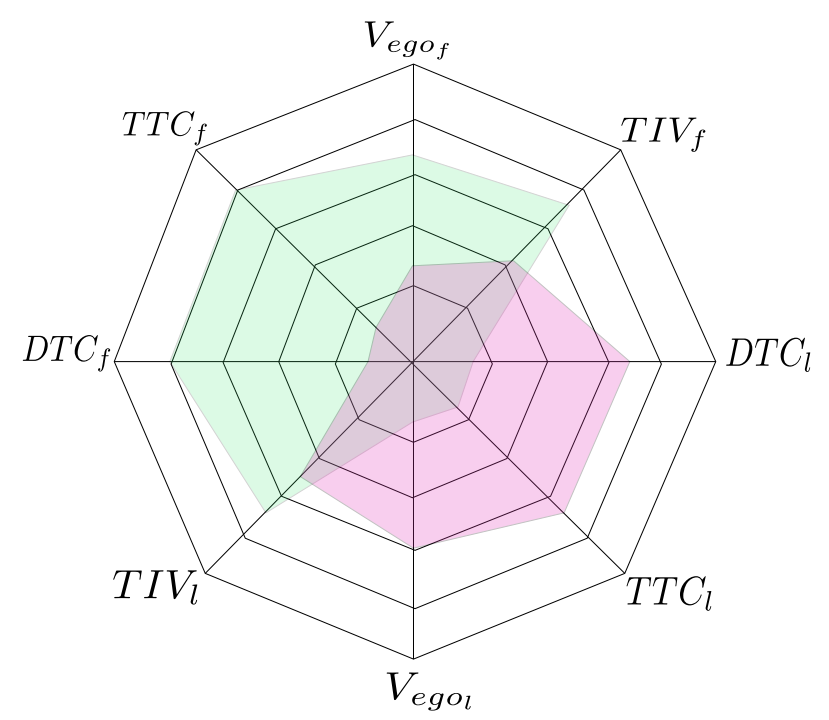

Fig. 2: Risk evaluation. Where $V_{\text {egof }}$ and $V_{\text {egol }}$ relative speed between ego vehicle and respectively front and lateral vehicles, $D T C_{f}$ and $D T C_{l}$ are distance to collision between ego vehicle and respectively front and lateral vehicles, $T T C_{f}, T T C_{f}$ are time to collision front and lateral vehicles and $T I V_{f}, T I V_{f}$ are intervehicle time in front and lateral respectively

When a decision can be formulated based on a relationship between mutual information, mutual dependence can be deduced. The mutual information between two discrete vectors is given as follows:

$$
m_{i}(X, Y)=\sum_{y \in Y} \sum_{x \in X} p(x, y) \log \left(\frac{p(x, y)}{p(x) p(y)}\right)
$$


where $p(x), p(y)$ are the marginal probability distribution function of $x$ and $y$ respectively, and $p(x, y)$ is the joint probabilistic distribution of $x$ and $y$.

Moreover, Hybrid state system and hidden Markov models are mixed to estimate driver behaviour and the unknown decision of other vehicle [12]. Another approach based on classification and regression trees has been established in order to predict and estimate driving manoeuvre risks.

However, the accurate calculation of mutual dependence is hard to achieve because numerous external indicators are not available. To design and improve functional safety of autonomous vehicle, this article proposes a machine learning based simulation approach.

\section{B. Adaboost based Method}

This section introduces the Adaboost classification algorithm [21]. It is suitable for binary classification in which a strong classifier is achieved based on iterative minimization of exponential loss criteria and linear combination of weak classifiers.

Let $\Gamma=\left\{\left(x_{i}, y_{i}\right), i=\overline{1, n}\right\}$ denote a train-

ing set of $n$ independent observations, where $x_{i}=$ $\left(x_{i, 1}, x_{i, 2}, \ldots, x_{i, p}\right)^{T} \in \mathbb{R}^{p}$ represents the input attributes for manoeuvre decision. $y_{i} \in\{+1,-1\}$ the class label of the $i$ th instance, where the finite discrete values " +1 " and " $-1 "$ are respectively the succeeded and failed labelled manoeuvre.

Input:

Let consider $\left(x_{i}, y_{i}\right), i=\overline{1, n}$ set of $n$ observation or training sample data, and $H=\left\{h_{k}\right\}$ the set of constructed weak classifiers.

Weak classifier: Classification And Regression Tree $h: x \rightarrow[1,-1]$

Initial weights: $w_{i}^{(1)}=\frac{1}{n}$ for $i=\overline{1, n}$ such that $\sum_{i=1}^{n} w_{i}^{(1)}=1$. As there are as much successes as failures, it is not necessary to take adjustable initial weights.

Let $m$ be the maximum number of iterations

For $j=\overline{1, m}$ do

Let $\varepsilon_{j}$ be the sum of weighed weak classifiers $h_{j}$ :

$\varepsilon_{j}=\sum_{i=1}^{n} w_{i}^{j} I\left[y_{i} \neq h_{j}\left(x_{i}\right)\right]$

$I[\gamma]=\left\{\begin{array}{cc}1 & \gamma=\text { Succeeded } \\ 0 & \gamma=\text { failed }\end{array}\right.$

Generate weak classifiers $h_{k}$ such that $\varepsilon_{k}$ the sum of weighed weak classifiers is minimizing: $h^{j}=\arg \min _{h^{j} \in H} \varepsilon^{j}$

where

$$
\begin{aligned}
& \varepsilon_{j}=\sum_{i=1}^{n} w_{i}^{j} I\left[y_{i} \neq h_{j}\left(x_{i}\right)\right] \text { and } I[\gamma]= \\
& \left\{\begin{array}{cc}
1 & \gamma=\text { Succeeded } \\
0 & \gamma=\text { failed }
\end{array}\right.
\end{aligned}
$$

Learning coefficient $\alpha^{j}=\frac{1}{2} \ln \left(\frac{1-\varepsilon^{j}}{\varepsilon^{j}}\right)$ of each weak classifier

Update weights distribution $w_{i}^{j+1}=w_{i}^{j} e^{-\alpha^{j} y_{i} h^{j}\left(x_{i}\right)}$ Renormalize $D_{i}^{j+1}=w_{i}^{j+1} / S_{j}$ for $\forall i$, where $S_{j}=$ $\sum_{i=1}^{n} w_{i}^{j} e^{-\alpha^{j} y_{i} h^{j}\left(x_{i}\right)}$

end for

Output: The final strong classifier:

$H=\operatorname{sign}\left(\sum_{j=1}^{m} \alpha^{j} h^{j}(x)\right)$

In the next section, we will combine the elements described above to carry out a method enabling safe decision making during driverless manoeuvre.

\section{FRAMEWORK FOR LEARNING-BASED DECISION MAKING}

Many important social and physical phenomena can be numerically modelled allowing an accurate representation of the real world. Furthermore, through simulation it is possible to quantify performance and provide relevant metrics about the considered model. In the case of ADS development and scenario-based testing, simulation enables deeper investigation of common scenarios and also situations which may not be very frequent on the roads. Simulation also helps in training and testing the system behaviour in critical and edge case scenarios. Therefore, simulation represents a powerful source of feedback and is a relevant means of validation.

The proposed approach uses SCANeR-studio which provides a framework for performing the testing and the analysis of autonomous driving systems in a virtual environment. It can be run on a desktop pc. The simulation environment contains models of cameras and radars for perception systems, models of both ego and agent vehicles, a model for the driver and models of physical environment (weather, light, infrastructures, agents, etc.). The simulator feeds sensor data to control the system which executes a path planning algorithm.

To validate the proposed approach, we consider to collect simulation data in a Cut-In scenario. 


\section{A. Data collection}

A Cut-In manoeuvre, as illustrated in Fig. 3, consists in changing lanes by moving in front of or in between the vehicles that are driving in the target lane. For this scenario, depending on the number of vehicles in the target lane and the gap between those vehicles, numerous possibilities and test cases can be considered, taking into account increase and decrease of speed, reduced space, visibility, etc. The parameters considered for this scenario are as follows:

$\begin{array}{lc}\text { Light } & \text { day light } \\ \text { Weather } & \text { sunny } \\ \text { Jay-walking } & \text { no } \\ \text { Parked vehicle } & \text { no } \\ \text { Speed of ego vehicle } & {[10,100] \mathrm{km} / \mathrm{h}} \\ \text { Speed of agent } & {[10,70] \mathrm{km} / \mathrm{h}}\end{array}$

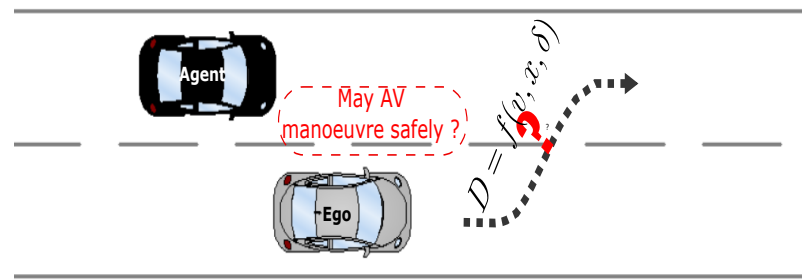

Fig. 3: Cut-In manoeuvre.

Simulation proceed iteratively by varying speed of both the ego vehicle and the agent vehicle. At each run of the simulation as shown in Fig. 4, metrics for the test case are measured (TIV, DIV, TTC and DTC). This process is repeated thousand times to cover representative parameter ranges. In this study we consider that thousands of simulations are sufficient. Furthermore having more simulation and more data can only improve the result.

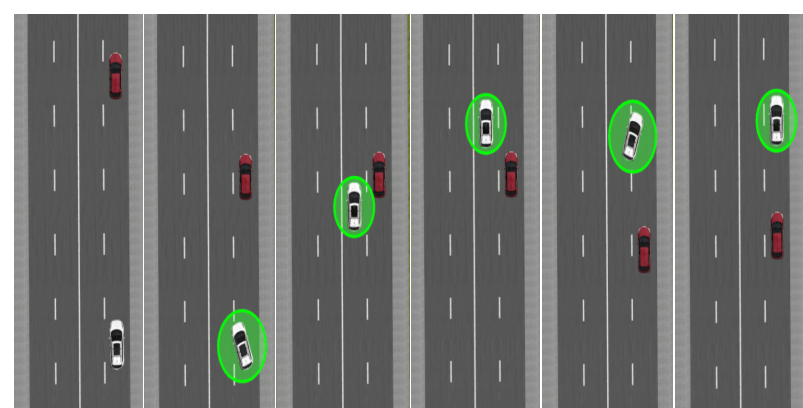

Fig. 4: Scenario data collection.

\section{B. Simulation results}

We present results from the application of proposed framework to the cut-in scenario. Thousands of simulations are executed. A part of collected data is used for the training and the remaining part is used for the validation. To implement the Adaboost classifier we chose as input the ego vehicle speed and the agent speed which are relevant variables for a collision, and as output the distance to collision (DTC).

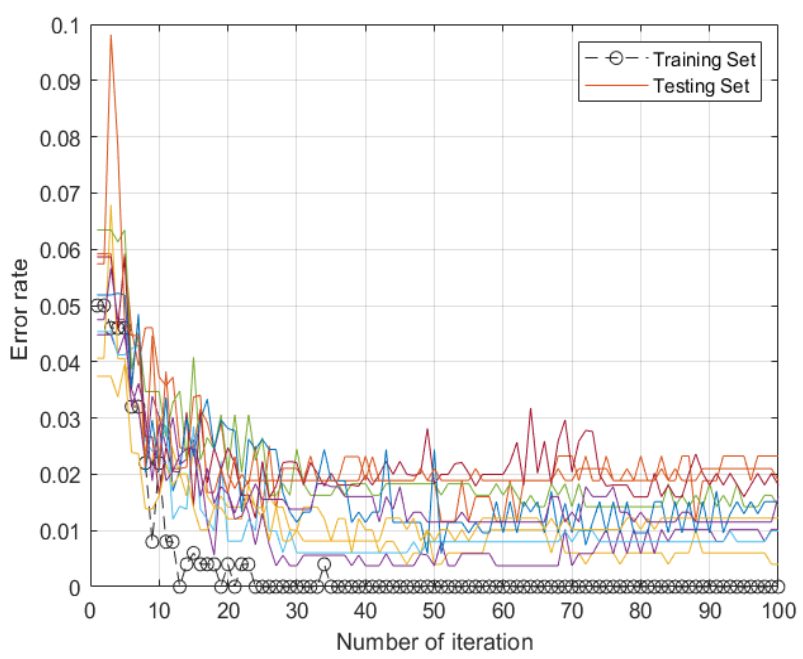

Fig. 5: Learning validation error.

As shown in Fig. 5, hundred of classifications are executed with a random selection of training data set. Fig. 5 illustrates that whatever the chosen training set is, the validation error converges to a limit under the threshold of $2 \%$. Therefore, such machine learning approach can be considered robust and suitable for scenarios that involve uncertainty and unknown decisions of others vehicles such as braking or overtaking.

In Fig. 6 we represent the simulated data by the red colour, the red-point represents the failed manoeuvres and the red-plus represents the succeeded manoeuvre. The green circle represents the prediction of failed manoeuvre, whereas the blue square represents the prediction of succeeded manoeuvre.

Fig. 6 shows that, through the proposed framework, we can predict the result of manoeuvres which is helpful to make dynamic decision in a driverless state enabling to avoid dangerous manoeuvres. Indeed, the results demonstrate that we can identify manoeuvres that violate a safety goal of no collision.

Moreover, Fig. 6 shows that machine learning could be performed by simulation since the tests proved that the proposed method could predict the critical 
situations. The results show that a simulation-based machine learning is an efficient way to manage the different scenarios faced by $\mathrm{AV}$ and that this machine learning approach can improve substantially the decision making.

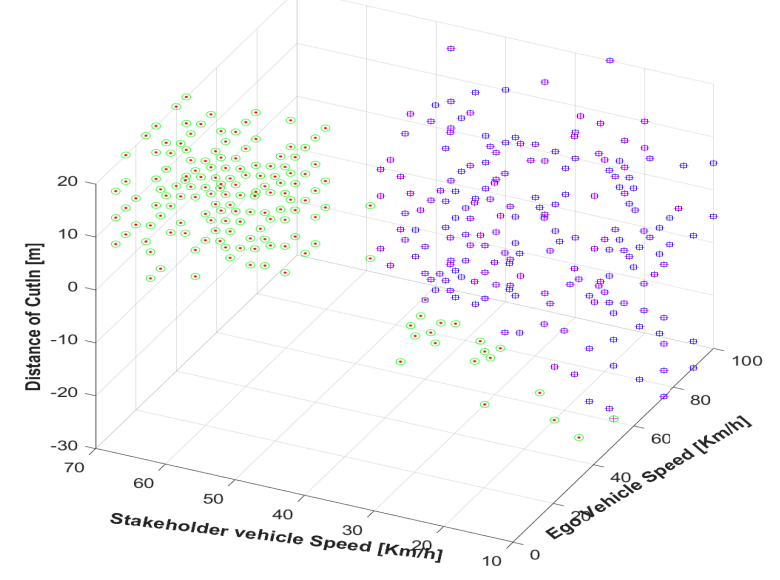

Fig. 6: Cut-in criticality prediction.

\section{CONClusion}

Although it is hard to foresee the future, we can imagine that autonomous vehicles will be a part of it when the regulatory and technical challenges are solved. This paper proposes a simulation-based machine learning approach enabling ADS to learn how to make a safe decision before or during manoeuvre. The proposed approach uses the Adaboost classifier which has been trained using experimental data generated by a virtual test driver. A remarkable ability to predict the simulation outcome is shown.

\section{REFERENCES}

[1] S. international, "Taxonomy and definitions for terms related to driving automation systems for on-road motor vehicles," SAE International, (J3016), 2018.

[2] L. Li, K. Ota, and M. Dong, "Humanlike driving: Empirical decision-making system for autonomous vehicles," IEEE Transactions on Vehicular Technology, vol. 67, no. 8, pp. 6814-6823, 2018.

[3] N. Kalra and S. M. Paddock, "Driving to safety: How many miles of driving would it take to demonstrate autonomous vehicle reliability?" Transportation Research Part A: Policy and Practice, vol. 94, pp. 182-193, 2016.

[4] H. Cheng, Autonomous intelligent vehicles: theory, algorithms, and implementation. pringer Science \& Business Media, 2011.

[5] M. Maurer, J. C. Gerdes, B. Lenz, H. Winner et al., Autonomous driving. Springer, 2016.
[6] J. Van Brummelen, M. O’Brien, D. Gruyer, and H. Najjaran, "Autonomous vehicle perception: The technology of today and tomorrow," Transportation research part C: emerging technologies, 2018.

[7] M. Abe, Vehicle handling dynamics: theory and application. Butterworth-Heinemann, 2015.

[8] C. Hatipoglu, U. Ozguner, and K. A. Redmill, "Automated lane change controller design," IEEE transactions on intelligent transportation systems, vol. 4, no. 1, pp. 13-22, 2003.

[9] J. Feng, J. Ruan, and Y. Li, "Study on intelligent vehicle lane change path planning and control simulation," in IEEE International Conference on Information Acquisition. IEEE, 2006, pp. 683-688.

[10] J. E. Naranjo, C. Gonzalez, R. Garcia, and T. De Pedro, "Lane-change fuzzy control in autonomous vehicles for the overtaking maneuver," IEEE Transactions on Intelligent Transportation Systems, vol. 9, no. 3, p. 438, 2008.

[11] L. Claussmann, A. Carvalho, and G. Schildbach, "A path planner for autonomous driving on highways using a human mimicry approach with binary decision diagrams," in Control Conference (ECC), 2015 European. IEEE, 2015, pp. 29762982.

[12] V. Gadepally, A. Krishnamurthy, and U. Ozguner, "A framework for estimating driver decisions near intersections," IEEE Transactions on Intelligent Transportation Systems, vol. 15, no. 2, pp. 637-646, 2014.

[13] M. Acosta and S. Kanarachos, "Teaching a vehicle to autonomously drift: A data-based approach using neural networks," Knowledge-Based Systems, vol. 153, pp. 12-28, 2018.

[14] N. Li, D. W. Oyler, M. Zhang, Y. Yildiz, I. Kolmanovsky, and A. R. Girard, "Game theoretic modeling of driver and vehicle interactions for verification and validation of autonomous vehicle control systems," IEEE Transactions on control systems technology, vol. 26, no. 5, pp. 1782-1797, 2018.

[15] T. Rosenstatter and C. Englund, "Modelling the level of trust in a cooperative automated vehicle control system," IEEE Transactions on Intelligent Transportation Systems, vol. 19, no. 4, pp. 1237-1247, 2018.

[16] L. Claussmann, M. O’Brien, S. Glaser, H. Najjaran, and D. Gruyer, "Multi-criteria decision making for autonomous vehicles using fuzzy dempster-shafer reasoning," in 2018 IEEE Intelligent Vehicles Symposium (IV). IEEE, 2018, pp. 2195-2202.

[17] A. Furda and L. Vlacic, "Enabling safe autonomous driving in real-world city traffic using multiple criteria decision making," IEEE Intelligent Transportation Systems Magazine, vol. 3, no. 1, pp. 4-17, 2011.

[18] H. Chae, Y. Jeong, S. Kim, H. Lee, J. Park, and K. Yi, "Design and vehicle implementation of autonomous lane change algorithm based on probabilistic prediction," in 2018 21st International Conference on Intelligent Transportation Systems (ITSC). IEEE, 2018, pp. 2845-2852.

[19] H. Chae, K. C. Min, and K. Yi, "Model predictive control based automated driving lane change control algorithm for merge situation on highway intersection," SAE Technical Paper, Tech. Rep., 2017.

[20] J. C. Hayward, "Near miss determination through use of a scale of danger," 1972.

[21] Y. Freund and R. E. Schapire, "A decision-theoretic generalization of on-line learning and an application to boosting," Journal of computer and system sciences, vol. 55, no. 1, pp. 119-139, 1997. 